\title{
Effect of Electrostriction on the Self-organization of Porous Nanostructures in Anodized Aluminum Oxide
}

\author{
L. G. Stanton ${ }^{1}$ and A. A. Golovin \\ Department of Applied Mathematics, Northwestern University, 60208 Evanston, USA
}

\begin{abstract}
The self-organization of porous nanostructures in anodic metal oxide is considered. A mathematical model which incorporates the chemical reactions at the metal-oxide and oxide-electrolyte interfaces and elastic stress caused by the electrostrictive effects is developed. It is shown through linear stability analysis, that a short-wave instability exists in certain parameter regimes which can lead to the formation of hexagonally ordered pores observed in anodized aluminum oxide.
\end{abstract}

Key words: electrostriction, nanostructures, stability analysis

AMS subject classification: $78 \mathrm{~A} 35,35 \mathrm{P} 15$

\section{Introduction}

During the process of anodization in acidic electrolytes, some metals will form a uniformly thick oxide barrier layer on the surface of the anode. As the applied voltage to the anode is increased, the thickness of this barrier layer will in turn linearly increase with the voltage [1, ch.6]. In certain metals, such as aluminum [2, 3], titanium $[4,5,6]$, zirconium [7], niobium [8], tantalum [9], tungsten [10] and tin [11], the surface of the barrier layer can become unstable, causing pores to form in the oxide. These nanostructured materials have found applications in many areas such as catalysts, gas sensors and coatings for batteries. In particular, aluminum has parameter regimes in which these pores will self-assemble in hexagonally ordered arrays as shown in Fig. $1[12,13,14,15]$. These pores can have aspect ratios as high as 1:1000 with diameters of the order 10-100 $\mathrm{nm}$ [16].

Anodized aluminum oxide (AAO) has vast scientific and industrial applications as well. In the past, $\mathrm{AAO}$ has been used to greatly improve the surface properties of aluminum $[1,17]$, and recently, due to the pore sizes and array regularity, it has gained great interest

\footnotetext{
${ }^{1}$ Corresponding author. E-mail: l-stanton@northwestern.edu
} 


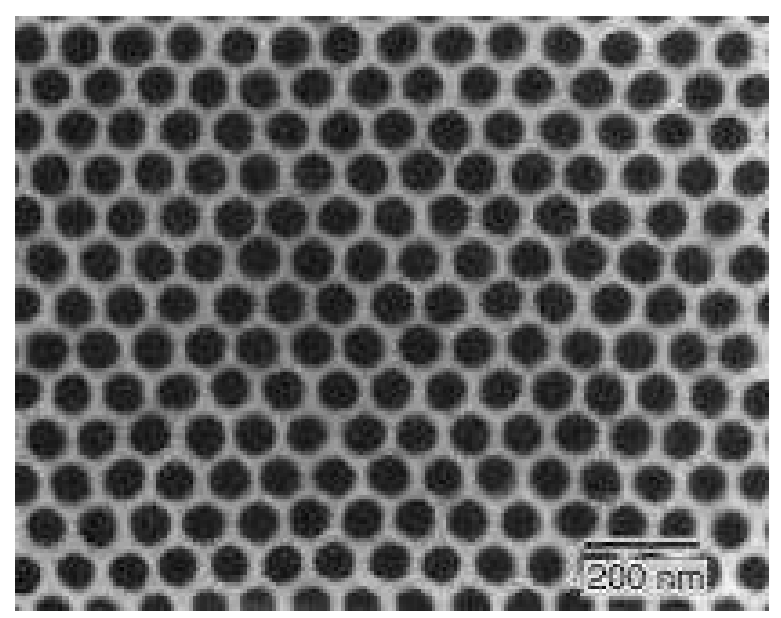

Figure 1: SEM micrograph of AAO hole configuration. Reprinted in part from Ref. [12] with permission of AAAS (1995) (C).

as a template for the fabrication of many nanoscale particles such quantum dots, nanowires and nanotubes $[18,19,20,21]$. While many experimental advancements have been made in the last few decades to refine AAO production [22], the theoretical understanding of porous AAO formation is still in its infancy.

In $[23,24]$, the steady growth of a single pore was studied, and a long-wave linear stability analysis was performed on a similar model in [25]. Works such as [25, 26, 27], however, lack any physical mechanism to damp short-wave disturbances, and in [28], the surface energy effects induced by the deformable interfaces were proposed as a possible mechanism to provide this short-wave cutoff. In [29], the effects of interfacial diffusion at the metal-oxide interface were investigated, and in [30], the effects of a field-dependent oxide conductivity were explored. The model proposed by Singh et al. in [31, 32] included the contributions of elastic stress to the surface activation energies, and it was shown that these effects can result in a short-wave instability which can explain the formation of hexagonally ordered pores. The validity of this model was explored experimentally in [33, 34, 35, 36].

In this work, we propose an alternative elastic effect to that studied in [31, 32]. While Singh et al. examined elastic effects resulting from epitaxial stress at the metal-oxide interface, we will instead consider the effect of electrostriction induced by the electric field as a possible mechanism for pattern formation. Electrostrictive effects have been suggested before as a possible mechanism for the initial pattern selection during anodization [37], but a thorough analysis of any mathematical model has yet to be carried out. We will additionally use the Butler-Volmer relations to model charge flux at the metal-oxide and oxide-electrolyte interfaces as well the stress contributions to the activation energies at these surfaces. We use linear stability analysis on a basic state solution of our system to show that electrostriction can indeed lead to a short-wave instability, which is necessary for the self-assembly of ordered porous arrays. We also present a dispersion relation which can be used to predict typical pore diameters and spacings along with phase diagrams which predict parameter regimes for 
barrier-type, ordered-porous and disordered-porous oxides. As aluminum oxide can exhibit barrier layers as well as both ordered and disordered porous arrays, we will assume the anode to be aluminum, although the analysis can be easily generalized to other substrate materials.

\section{Problem Formulation}

We begin by analyzing the effects of the electric field $\mathbf{E}$ on the deformations within the oxide layer. The oxide can support electric fields of the order $|\mathbf{E}| \sim 10^{9} \mathrm{~V} / \mathrm{m}[1$, ch.6], and as the electrostrictive coefficients of $\mathrm{Al}_{2} \mathrm{O}_{3}$ have been observed to be of the order $10^{-21} \mathrm{~m}^{2} / \mathrm{V}^{2}$ [38], we expect strains to be around $0.1 \%$. These deformations will destroy the isotropy of the dielectric permeability $\epsilon_{0}$ and replace this scalar quantity with the rank-2 tensor $\epsilon_{i j}$. The state of the deformed layer can be described by the linearized strain tensor with the kinematic relationship

$$
u_{i j} \equiv \frac{1}{2}\left(\partial_{j} u_{i}+\partial_{i} u_{j}\right), \quad i, j=\{1,2,3\}
$$

where the operator $\partial_{i}$ denotes the partial derivative with respect to the $i^{\text {th }}$ spatial coordinate, and the elements $u_{i}$ are components of the displacement vector $\mathbf{u}$. As these quantities will be typically small, the perturbations to the dielectric permeability can be truncated to first order. The most general form for the adjusted dielectric tensor of an isotropic body is hence

$$
\epsilon_{i j}=\epsilon_{0} \delta_{i j}+a_{1} u_{i j}+a_{2} u_{k k} \delta_{i j}
$$

where $\delta_{i j}$ is the Kronecker delta, and Einstein notation is used for the summation over repeated indices. From this expression, it can then be shown that the corresponding stress tensor becomes

$$
\sigma_{i j}=\sigma_{i j}^{(0)}+\alpha E_{i} E_{j}-\beta|\mathbf{E}|^{2} \delta_{i j}, \quad \alpha=\frac{2 \epsilon_{0}-a_{1}}{8 \pi}, \quad \beta=\frac{\epsilon_{0}+a_{2}}{8 \pi},
$$

where $\sigma_{i j}^{(0)}$ is the stress tensor in the absence of the electric field [39]. Using the elastic moduli of $\mathrm{Al}_{2} \mathrm{O}_{3}$ and the above assumptions about the magnitude of the strain, we expect $\alpha$ and $\beta$ to be of the order $10^{-9} \mathrm{~N} / \mathrm{V}^{2}$. We can use Hooke's law to write this tensor in terms of the strain as

$$
\sigma_{i j}^{(0)}=2 \mu\left[u_{i j}+\left(\frac{\nu}{1-2 \nu}\right) u_{k k} \delta_{i j}\right]
$$

where $\mu$ is the shear modulus and $\nu$ is the Poisson ratio. We set the $z$-coordinate to be perpendicular to the initially planar anode surface and represent the metal-oxide (MO) and oxide-electrolyte $(\mathrm{OE})$ interfaces as $z=h_{1}(\mathbf{x}, t)$ and $z=h_{2}(\mathbf{x}, t)$ respectively with $\mathbf{x}=(x, y)$ as shown in Fig. 2.

As the thickness of the anode is on a far larger scale than typical pore dimensions, we will approximate the metal substrate to be infinitely deep. Within both the metal anode 


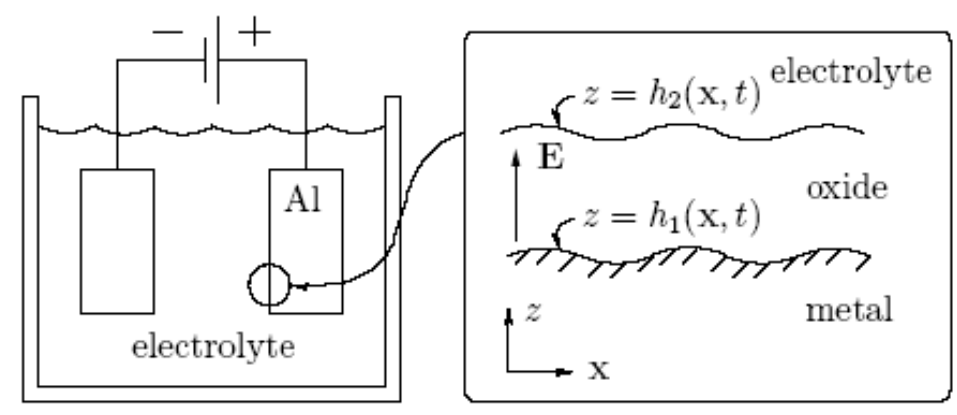

Figure 2: Schematic of interfaces at anode surface. Note that the domain has been rotated.

and the oxide film, mechanical equilibrium can then be appropriately modeled by the Navier equation

$$
\partial_{j} \sigma_{i j}^{m, o}=0 \text { for } i=\{1,2,3\}
$$

where the superscripts $(m, o)$ denote the metal and oxide phases respectively. Note that the electric field is not present in the metal substrate. Furthermore, in writing the charge flux (or current density) of the oxide layer in terms of the field potential as $\mathbf{j}=\sigma_{0} \mathbf{E}=-\sigma_{0} \nabla \varphi$ and assuming the conductivity $\sigma_{0}$ to be constant, the conservation of charge, $\nabla \cdot \mathbf{j}=0$, reduces to the Laplace equation for the potential

$$
\nabla^{2} \varphi=0
$$

The deformation vector components $u_{i}^{m, o}$ will then satisfy

$$
\begin{array}{cl}
\left(1-2 \nu^{m}\right) \nabla^{2} \mathbf{u}^{m}+\nabla\left(\nabla \cdot \mathbf{u}^{m}\right)=0 & \text { for } z \in\left(-\infty, h_{1}\right), \\
\mu^{o} \nabla^{2} \mathbf{u}^{o}+\left(\frac{\mu^{o}}{1-2 \nu^{o}}\right) \nabla\left(\nabla \cdot \mathbf{u}^{o}\right)=\beta \nabla\left(|\nabla \varphi|^{2}\right)-\alpha(\nabla \varphi \cdot \nabla) \nabla \varphi & \text { for } z \in\left(h_{1}, h_{2}\right) .
\end{array}
$$

for $i=\{1,2,3\}$. With the bulk equations established for the metal substrate and oxide layer, we must next determine appropriate boundary conditions at the interfaces between the metal, oxide and electrolyte as well as far field conditions in the anode away from the oxide. Far from the MO interface, we will assume that any deformations in the metal due to the electrostriction of the oxide layer will decay, so we have

$$
\mathbf{u}^{m} \rightarrow 0 \text { as } z \rightarrow-\infty .
$$

At the MO interface, we will assume continuity in the deformations as well as a stress balance

$$
u_{i}^{m}=u_{i}^{o} \text { and }\left(\sigma_{i j}^{m}-\sigma_{i j}^{o}\right) n_{j}^{(1)}=0 \text { for } i=\{1,2,3\} \text { on } z=h_{1}(\mathbf{x}, t),
$$

where the outward normal $\mathbf{n}^{(1)}$ points into the oxide. At the OE interface, the liquid electrolyte will be assumed to be stress-free, so the stress balance at this interface becomes

$$
\sigma_{i j}^{o} n_{j}^{(2)}=0 \text { for } i=\{1,2,3\} \text { on } z=h_{2}(\mathbf{x}, t)
$$


with the normal $\mathbf{n}^{(2)}$ here pointing into the electrolyte. We can express these normal vectors in terms of the given interface shapes as

$$
\mathbf{n}^{(1,2)}=\frac{\left(-\partial_{x} h_{1,2},-\partial_{y} h_{1,2}, 1\right)}{\sqrt{1+\left|\nabla h_{1,2}\right|^{2}}} .
$$

To establish boundary conditions for $\varphi$, we first assume the potential at the MO interface to be set at the constant applied voltage

$$
\varphi=\mathcal{V} \text { on } z=h_{1}(\mathbf{x}, t)
$$

and at the OE interface, we have the charge flux balance:

$$
j_{n}=-\sigma_{0} \frac{\partial \varphi}{\partial n}=K_{+}-K_{-} \text {on } z=h_{2}(\mathbf{x}, t) .
$$

Here, $j_{n}$ is the normal component of the charge flux, and $K_{ \pm}$are the rates of the forward $(+)$ and reverse $(-)$ oxide dissolution reactions. One can use the Butler-Volmer relations to express these rates as

$$
K_{ \pm}=k_{ \pm} \exp \left\{ \pm\left(\eta \varphi+s_{i j}^{ \pm} \sigma_{i j}-\gamma^{ \pm} \kappa\right)\right\}
$$

where $k_{ \pm}$are the reaction rate constants for a planar interface in the absence of the electric field or stresses. The constant $\eta=q_{e} /\left(2 k_{B} T\right)$, where $q_{e}$ is the electron charge, $k_{B}$ is the Boltzmann constant, and $T$ is the absolute temperature. The symmetry factor has been taken to be $1 / 2$ for simplicity, which has been observed in many systems [40]. The activation surface energies are $\gamma^{ \pm}=\left(\partial E_{a}^{ \pm} / \partial \kappa\right) /\left(k_{B} T\right)$, the activation strains are $s_{i j}^{ \pm}=\left(\partial E_{a}^{ \pm} / \partial \sigma_{i j}\right) /\left(k_{B} T\right)$ and $E_{a}^{ \pm}$are the activation energies for the forward $(+)$and reverse $(-)$reactions of the planar OE interface. Note that $\gamma^{ \pm}$and $s_{i j}^{ \pm}$have been rescaled with the thermal energy, $k_{B} T$, and the mean interfacial curvature is given by $\kappa=-(1 / 2) \nabla \cdot \mathbf{n}$. We will include the effect of the electrolyte $\mathrm{pH}$ on the oxide dissolution as $k_{+}=k_{+}^{0} 10^{-p H}$ [23]. To complete our model, we prescribe conditions for the normal velocities

$$
v_{n}=\frac{\partial_{t} h}{\sqrt{1+|\nabla h|^{2}}}
$$

at each interface. We will assume that the evolution of each interface will be determined by the electrochemical reactions that sustain the current in the electrolytic cell which gives

$$
\begin{array}{ll}
v_{n}=-a \sigma_{0} \frac{\partial \varphi}{\partial n} & \text { on } z=h_{1}(\mathbf{x}, t), \\
v_{n}=-b_{+} K_{+}+b_{-} K_{-} & \text {on } z=h_{2}(\mathbf{x}, t) .
\end{array}
$$

The constants $a, b_{ \pm}$are Faradaic coefficients relating the interfacial currents to the rate of the interface motion. 


\section{Basic State}

We start by calculating a basic state of the system, where we use bars to denote basic state solutions. We assume an oxide layer of barrier type which implies interfaces that are planar and hence only depend on time $h_{1,2}=\bar{h}_{1,2}(t)$. In the metal substrate, we have

$$
\overline{\mathbf{u}}^{m}=0
$$

which consequently gives $\bar{\sigma}_{i j}^{m}=\bar{u}_{i j}^{m}=0$. In the oxide layer, the potential and deformations are linear in $z$

$$
\bar{\varphi}=\mathcal{V}-E\left(z-\bar{h}_{1}\right), \quad \overline{\mathbf{u}}^{o}=\left(0,0, \bar{u}_{33}^{o}\left(z-\bar{h}_{1}\right)\right),
$$

where $E$ is the electric field. The stress and strain tensors are constant, and the only nonzero elements are given as

$$
\bar{u}_{33}^{o}=\frac{E^{2}\left(1-2 \nu^{o}\right)}{2 \mu^{o}\left(1-\nu^{o}\right)}(\beta-\alpha), \quad \bar{\sigma}_{11}^{o}=\bar{\sigma}_{22}^{o}=\frac{-E^{2}}{\left(1-\nu^{o}\right)}\left[\nu^{o} \alpha+\left(1-2 \nu^{o}\right) \beta\right] .
$$

Note that this merely renormalizes the rate constants

$$
\bar{k}_{ \pm}=k_{ \pm} \exp \left\{ \pm\left[\eta \mathcal{V}+\left(s_{11}^{ \pm}+s_{22}^{ \pm}\right) \bar{\sigma}_{11}^{o}\right]\right\}
$$

The remaining boundary conditions give a system for the field $E$ and the layer thickness $L=h_{2}-h_{1}$

$$
\begin{aligned}
\sigma_{0} E & =\bar{k}_{+} e^{-\eta E L}-\bar{k}_{-} e^{\eta E L}, \\
\frac{d L}{d t} & =a \sigma_{0} E-b_{+} \bar{k}_{+} e^{-\eta E L}+b_{-} \bar{k}_{-} e^{\eta E L}
\end{aligned}
$$

This system will always relax to a steady state under the sufficient conditions $a>b_{ \pm}$which gives

$$
E=\sigma_{0}^{-1}\left(\bar{k}_{+} \bar{k}_{-}\right)^{1 / 2}\left(r-r^{-1}\right), \quad L=\frac{\sigma_{0} \ln \left[\bar{k}_{+} /\left(\bar{k}_{-} r^{2}\right)\right]}{2 \eta\left(\bar{k}_{+} \bar{k}_{-}\right)^{1 / 2}\left(r-r^{-1}\right)}, \quad r=\sqrt{\frac{a-b_{-}}{a-b_{+}}},
$$

and this oxide layer of constant thickness $L$ will propagate with the uniform velocity $v=$ $a \sigma_{0} E$ into the substrate. For physically relevant solutions, we must additionally have $b_{+}>b_{-}$ for $E>0$, and $\bar{k}_{+}>\bar{k}_{-} r^{2}$ for $L>0$. Note that this barrier layer thickness scales linearly with the voltage as seen in experiments [1, ch.6]. See [32] for a more detailed analysis of a basic state solution in this form. This steady state corresponds to a barrier layer in which the dissolution of the oxide into the electrolyte is balanced by the oxidation of the anode, and it is upon this state which we will perform a linear stability analysis. 


\section{Linear Stability Analysis}

We now examine the linear stability of the basic state solution. For convenience, we change to the moving coordinate frame with speed $v$ in the negative $z$-direction and set the origin to be coincident with the MO interface. In this frame,

$$
\bar{h}_{1}=0, \quad \bar{h}_{2}=L, \quad \bar{\varphi}=\mathcal{V}-E z, \quad \overline{\mathbf{u}}^{o}=\left(0,0, \bar{u}_{33}^{o} z\right)
$$

while the other basic state solutions remain unchanged. We next perturb these solutions with the infinitesimal quantities

$$
h_{1,2}=\bar{h}_{1,2}+\tilde{h}_{1,2}, \quad \mathbf{u}^{m, o}=\overline{\mathbf{u}}^{m, o}+\tilde{\mathbf{u}}^{m, o}, \quad \varphi=\bar{\varphi}+\tilde{\varphi}
$$

which in turn perturb the tensors $u_{i j}^{m, o}$ and $\sigma_{i j}^{m, o}$. These perturbations take the linearized form

$$
\begin{aligned}
\tilde{u}_{i j}^{m, o} & =\frac{1}{2}\left(\partial_{j} \tilde{u}_{i}^{m, o}+\partial_{i} \tilde{u}_{j}^{m, o}\right), \quad \tilde{\sigma}_{i j}^{m}=2 \mu^{m}\left[\tilde{u}_{i j}^{m}+\left(\frac{\nu^{m}}{1-2 \nu^{m}}\right) \tilde{u}_{k k}^{m} \delta_{i j}\right], \\
\tilde{\sigma}_{i j}^{o} & =2 \mu^{o}\left[\tilde{u}_{i j}^{o}+\left(\frac{\nu^{o}}{1-2 \nu^{o}}\right) \tilde{u}_{k k}^{o} \delta_{i j}\right]+2 \beta E \partial_{z} \tilde{\varphi} \delta_{i j}-\alpha E\left(\delta_{i 3} \partial_{j} \tilde{\varphi}+\delta_{3 j} \partial_{i} \tilde{\varphi}\right) .
\end{aligned}
$$

After linearizing the system, we obtain the governing equations for the deformation vector perturbation components $\tilde{u}_{i}^{m, o}$

$$
\begin{array}{cc}
\left(1-2 \nu^{m}\right) \nabla^{2} \tilde{\mathbf{u}}^{m}+\nabla\left(\nabla \cdot \tilde{\mathbf{u}}^{m}\right)=0 & \text { for } z \in(-\infty, 0), \\
\mu^{o} \nabla^{2} \tilde{\mathbf{u}}^{o}+\left(\frac{\mu^{o}}{1-2 \nu^{o}}\right) \nabla\left(\nabla \cdot \tilde{\mathbf{u}}^{o}\right)=(\alpha-2 \beta) E \nabla \tilde{\varphi}_{z} & \text { for } z \in(0, L),
\end{array}
$$

with the linearized boundary conditions

$$
\begin{array}{ll}
\tilde{u}_{i}^{m} \rightarrow 0 & \text { as } z \rightarrow-\infty, \\
\tilde{u}_{i}^{m}=\tilde{u}_{i}^{o}+\partial_{z} \bar{u}_{i}^{o} \tilde{h}_{1}, & \\
\tilde{\sigma}_{i 3}^{o}=\tilde{\sigma}_{i 3}^{m}+\bar{\sigma}_{i 1}^{o} \partial_{x} \tilde{h}_{1}+\bar{\sigma}_{i 2}^{o} \partial_{y} \tilde{h}_{1} & \text { at } z=0, \\
\tilde{\sigma}_{i 3}^{o}=\bar{\sigma}_{i 1}^{o} \partial_{x} \tilde{h}_{2}+\bar{\sigma}_{i 2}^{o} \partial_{y} \tilde{h}_{2} & \text { at } z=L,
\end{array}
$$

for $i=\{1,2,3\}$. The oxide potential perturbation, $\tilde{\varphi}$, is governed by

$$
\nabla^{2} \tilde{\varphi}=0 \text { for } z \in(0, L),
$$

with the linearized boundary conditions

$$
\begin{aligned}
\tilde{\varphi}= & E \tilde{h}_{1} \text { at } z=0, \\
\sigma_{0} \partial_{z} \tilde{\varphi}= & \left(A_{+}+A_{-}\right)\left(E \tilde{h}_{2}-\tilde{\varphi}\right)-\left(S_{i j}^{+}+S_{i j}^{-}\right) \tilde{\sigma}_{i j}^{o} \\
& +\left(\Gamma_{+}+\Gamma_{-}\right) \nabla^{2} \tilde{h}_{2} \text { at } z=L .
\end{aligned}
$$


For brevity, we have defined the constants

$$
A_{ \pm}=\eta \bar{k}_{ \pm} e^{\mp \eta E L}, \quad \Gamma_{ \pm}=\frac{1}{2} \gamma^{ \pm} \bar{k}_{ \pm} e^{\mp \eta E L}, \quad S_{i j}^{ \pm}=s_{i j}^{ \pm} \bar{k}_{ \pm} e^{\mp \eta E L}
$$

Finally, the evolution of interfacial perturbations is given by

$$
\begin{aligned}
\partial_{t} \tilde{h}_{1}= & a \sigma_{0} \partial_{z} \tilde{\varphi} \text { at } z=0, \\
\partial_{t} \tilde{h}_{2}= & \left(b_{+} A_{+}+b_{-} A_{-}\right)\left(E \tilde{h}_{2}-\tilde{\varphi}\right)-\left(b_{+} S_{i j}^{+}+b_{-} S_{i j}^{-}\right) \tilde{\sigma}_{i j}^{o} \\
& +\left(b_{+} \Gamma_{+}+b_{-} \Gamma_{-}\right) \nabla^{2} \tilde{h}_{2} \text { at } z=L .
\end{aligned}
$$

We next write the perturbations in the form

$$
\tilde{h}_{1,2}=\hat{h}_{1,2} \phi(\mathbf{x}, t), \quad \tilde{\mathbf{u}}^{m, o}=\hat{\mathbf{u}}^{m, o}(z) \phi(\mathbf{x}, t), \quad \tilde{\varphi}=\hat{\varphi}(z) \phi(\mathbf{x}, t),
$$

where the normal mode $\phi(\mathbf{x}, t)=\exp (\omega t+i \mathbf{q} \cdot \mathbf{x})$ with wave vector $\mathbf{q}$ and corresponding growth rate $\omega$. As the fields are isotropic, we take the wave vector $\mathbf{q}=(q, 0)$ without loss of generality, where $q>0$. This choice of wave vector decouples the fields $\hat{u}_{2}^{o, m}$ from the system, so we can solve for them separately. We are given

$$
\begin{gathered}
\left(\partial_{z}^{2}-q^{2}\right) \hat{u}_{2}^{m}=0 \text { for } z \in(-\infty, 0), \\
\left(\partial_{z}^{2}-q^{2}\right) \hat{u}_{2}^{o}=0 \text { for } z \in(0, L),
\end{gathered}
$$

with the boundary conditions

$$
\begin{aligned}
& \hat{u}_{2}^{m} \rightarrow 0 \quad \text { as } z \rightarrow-\infty, \\
& \hat{u}_{2}^{m}=\hat{u}_{2}^{o}, \quad \hat{\sigma}_{23}^{o}=\hat{\sigma}_{23}^{m} \quad \text { at } z=0 \text {, } \\
& \hat{\sigma}_{23}^{o}=0 \quad \text { at } z=L \text {. }
\end{aligned}
$$

After applying these boundary conditions, we see that the deformation perturbations in this direction take the trivial form

$$
\hat{u}_{2}^{m}=\hat{u}_{2}^{o}=0
$$

The remaining system of deformation eigenfunctions in the metal substrate are

$$
\begin{aligned}
& \left(1-2 \nu^{m}\right)\left(\partial_{z}^{2}-q^{2}\right) \hat{u}_{1}^{m}-q^{2} \hat{u}_{1}^{m}+i q \partial_{z} \hat{u}_{3}^{m}=0 \\
& \left(1-2 \nu^{m}\right)\left(\partial_{z}^{2}-q^{2}\right) \hat{u}_{3}^{m}+i q \partial_{z} \hat{u}_{1}^{m}+\partial_{z}^{2} \hat{u}_{3}^{m}=0
\end{aligned}
$$

for $z \in(-\infty, 0)$, and in the oxide layer,

$$
\begin{aligned}
& \mu^{o}\left(\partial_{z}^{2}-q^{2}\right) \hat{u}_{1}^{o}-\left(\frac{\mu^{o}}{1-2 \nu^{o}}\right)\left(q^{2} \hat{u}_{1}^{o}-i q \partial_{z} \hat{u}_{3}^{o}\right)=i q(\alpha-2 \beta) E_{0} \partial_{z} \hat{\varphi} \\
& \mu^{o}\left(\partial_{z}^{2}-q^{2}\right) \hat{u}_{3}^{o}+\left(\frac{\mu^{o}}{1-2 \nu^{o}}\right)\left(i q \partial_{z} \hat{u}_{1}^{o}+\partial_{z}^{2} \hat{u}_{3}^{o}\right)=(\alpha-2 \beta) E_{0} \partial_{z}^{2} \hat{\varphi}
\end{aligned}
$$


for $z \in(0, L)$. The boundary conditions are

$$
\begin{array}{ll}
\hat{u}_{k}^{m} \rightarrow 0 & \text { as } z \rightarrow-\infty, \\
\hat{u}_{k}^{m}=\hat{u}_{k}^{o}+\partial_{z} \bar{u}_{k}^{o} \hat{h}_{1}, & \\
\hat{\sigma}_{k 3}^{o}=\hat{\sigma}_{k 3}^{m}+i q \bar{\sigma}_{k 1}^{o} \hat{h}_{1} & \text { at } z=0, \\
\hat{\sigma}_{k 3}^{o}=i q \bar{\sigma}_{k 1}^{o} \hat{h}_{2} & \text { at } z=L,
\end{array}
$$

for $k=\{1,3\}$. The oxide potential eigenfunction $\hat{\varphi}$ is governed by

$$
\left(\partial_{z}^{2}-q^{2}\right) \hat{\varphi}=0
$$

for $z \in(0, L)$ with

$$
\begin{aligned}
\hat{\varphi}= & E \hat{h}_{1} \text { at } z=0, \\
\sigma_{0} \partial_{z} \hat{\varphi}= & \left(A_{+}+A_{-}\right)\left(E \hat{h}_{2}-\hat{\varphi}\right)-\left(\Gamma_{+}+\Gamma_{-}\right) q^{2} \hat{h}_{2} \\
& -\left(S_{i j}^{+}+S_{i j}^{-}\right) \hat{\sigma}_{i j}^{o} \text { at } z=L .
\end{aligned}
$$

The interfacial evolution is reduced to the algebraic equations

$$
\begin{aligned}
\omega \hat{h}_{1}= & a \sigma_{0} \partial_{z} \hat{\varphi} \text { at } z=0, \\
\omega \hat{h}_{2}= & {\left[\left(b_{+} A_{+}+b_{-} A_{-}\right) E-\left(b_{+} \Gamma_{+}+b_{-} \Gamma_{-}\right) q^{2}\right] \hat{h}_{2}-\left(b_{+} A_{+}+b_{-} A_{-}\right) \hat{\varphi} } \\
& -\left(b_{+} S_{i j}^{+}+b_{-} S_{i j}^{-}\right) \hat{\sigma}_{i j}^{o} \text { at } z=L .
\end{aligned}
$$

The general solution to the metal deformation problem can be found by letting $\hat{\mathbf{u}}^{m} \sim \mathbf{u}_{0} e^{\lambda z}$. This gives the corresponding characteristic equation

$$
2\left(1-2 \nu^{m}\right)^{2}\left(\lambda^{2}-q^{2}\right)^{2}=0,
$$

which has the degenerate solutions $\lambda= \pm q$. Each eigenvalue has a multiplicity of two and supports two linearly independent eigenvectors, so the general solution bounded at $z=-\infty$ is given as

$$
\hat{\mathbf{u}}^{m}=\mathbf{c}^{m} e^{q z}+\tilde{\mathbf{c}}^{m} z e^{q z},
$$

where the coefficient $\mathbf{c}^{m}$ is an arbitrary constant vector with elements $c_{1,2,3}^{m}$, and

$$
\tilde{\mathbf{c}}^{m}=q\left(\frac{c_{1}^{m}-i c_{3}^{m}}{3-4 \nu^{m}}, 0,-\frac{i c_{1}^{m}+c_{3}^{m}}{3-4 \nu^{m}}\right) .
$$

The general solution to the oxide deformation eigenfunction will have the same characteristic equation as Eq. (4.41) with the addition of a particular solution to account for the electric field induced inhomogeneity. We hence have

$$
\begin{aligned}
\hat{\mathbf{u}}^{o}= & \mathbf{c}^{o} \cosh (q z)+\mathbf{d}^{o} \sinh (q z)+\tilde{\mathbf{c}}^{o} z \cosh (q z)+\tilde{\mathbf{d}}^{o} z \sinh (q z) \\
& +\frac{1}{\mu^{o}}\left(1-2 \nu^{o}\right)(\alpha-2 \beta) E \hat{\varphi}\left[\begin{array}{lll}
0 & 0 & 1
\end{array}\right]^{T},
\end{aligned}
$$


where again the coefficients $\mathbf{c}^{o}$ and $\mathbf{d}^{o}$ are arbitrary constant vectors with elements $c_{1,2,3}^{o}$ and $d_{1,2,3}^{o}$ respectively. The remaining coefficients are given as

$$
\tilde{\mathbf{c}}^{o}=q\left(\frac{d_{1}^{o}-i c_{3}^{o}}{3-4 \nu^{o}}, 0,-\frac{i c_{1}^{o}+d_{3}^{o}}{3-4 \nu^{o}}\right), \quad \tilde{\mathbf{d}}^{o}=q\left(\frac{c_{1}^{o}-i d_{3}^{o}}{3-4 \nu^{o}}, 0,-\frac{i d_{1}^{o}+c_{3}^{o}}{3-4 \nu^{o}}\right) .
$$

If we then apply the deformation continuity conditions (4.31), the constants $c_{1,3}^{m}$ can be written as

$$
c_{1}^{m}=c_{1}^{o}, \quad c_{3}^{m}=c_{3}^{o}+\left[\bar{u}_{33}^{o}+\frac{E^{2}}{\mu^{o}}\left(1-2 \nu^{o}\right)(\alpha-2 \beta)\right] \hat{h}_{1} .
$$

After integrating the electric field equation (4.34) within the oxide and applying the voltage condition (4.35) at the MO interface, we can write the potential eigenfunction as

$$
\hat{\varphi}=E\left[\hat{h}_{1} \cosh (q z)+b \sinh (q z)\right]
$$

where $b$ is an arbitrary constant. The remaining boundary conditions are more complicated, so we express them as the system $\Lambda \mathbf{v}=\mathbf{0}$, where the vector of unknowns $\mathbf{v}=$ $\left(\hat{h}_{1}, \hat{h}_{2}, b, c_{1}^{o}, c_{3}^{o}, d_{1}^{o}, d_{3}^{o}\right)$. This matrix can be decomposed as $\boldsymbol{\Lambda}(\omega, q)=\boldsymbol{\Omega}(\omega)+\mathbf{Q}(q)$, and if we take the first two rows of $\Lambda$ to correspond to the the interfacial velocity conditions, then the nonzero elements of $\boldsymbol{\Omega}$ are given as

$$
\Omega_{11}=\Omega_{22}=\omega .
$$

We have listed the elements of $\mathbf{Q}$ in the appendix. For $\mathbf{v}$ to be nontrivial, the matrix $\boldsymbol{\Lambda}$ must be singular which gives the dispersion relation

$$
\operatorname{det}(\boldsymbol{\Lambda})=C_{2}(q) \omega^{2}+C_{1}(q) \omega+C_{0}(q)=0 .
$$

The coefficients $C_{i}(q)$ can be calculated as

$$
C_{0}=\operatorname{det}(\mathbf{Q}), \quad C_{1}=\operatorname{det}\left(\mathbf{Q}^{(1)}\right)+\operatorname{det}\left(\mathbf{Q}^{(2)}\right), \quad C_{2}=\operatorname{det}\left(\mathbf{Q}^{(1,2)}\right),
$$

where $\mathbf{Q}^{(i)}$ denotes the minor of $\mathbf{Q}$ generated by removing the $i^{\text {th }}$ row and column, while $\mathbf{Q}^{(i, j)}$ denotes the removal of both the $i^{\text {th }}$ and $j^{\text {th }}$ rows and columns.

\section{Results and Discussion}

The dispersion relation given by Eq. (4.50) has two roots, $\omega_{1,2}(q)$, which describe the growth rate of a mode with the wave number $q$. In the limit of spatially homogeneous perturbations $(q \rightarrow 0)$, these roots take the form

$$
\begin{array}{cl}
\omega_{1}(0)=0, \quad \omega_{2}(0)=-\frac{\sigma_{0} E^{2}\left[\left(a-b_{+}\right) A_{+}+\left(a-b_{-}\right) A_{-}\right]-\Sigma_{1}}{\sigma_{0} E+E L\left(A_{+}+A_{-}\right)-\Sigma_{2}} \\
\text { with } \quad \Sigma_{1}=2 \eta k_{+} k_{-} \bar{\sigma}_{11}^{o} E\left(b_{+}-b_{-}\right)\left(s_{11}^{+}-s_{11}^{-}+s_{22}^{+}-s_{22}^{-}\right), \\
\Sigma_{2}=2 \bar{\sigma}_{11}^{o}\left(S_{11}^{+}+S_{11}^{-}+S_{22}^{+}+S_{22}^{-}\right) .
\end{array}
$$




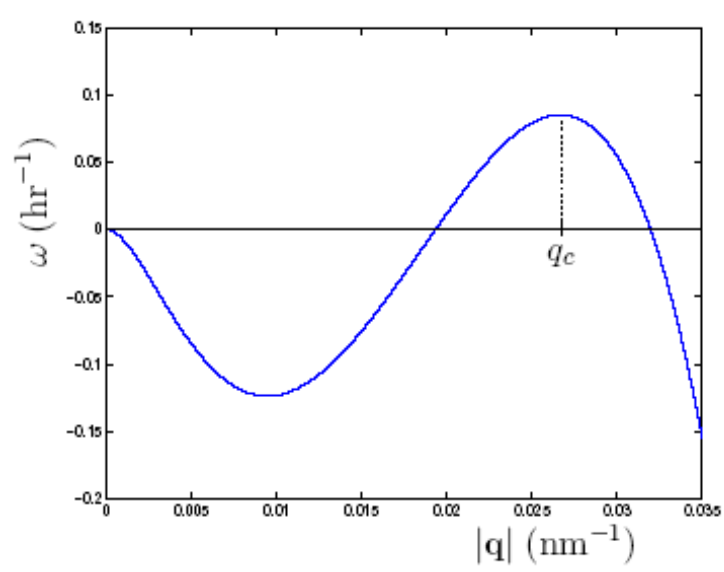

Figure 3: Dispersion relation yielding a short-wave instability. Parameters are taken as $\mathcal{V}=50 \mathrm{~V}, \sigma_{0}=5 \times 10^{-9} \mathrm{~A} / \mathrm{mV}, \eta=15.56 \mathrm{~V}^{-1}, \mathrm{pH}=0, k_{+}^{0}=35 \mathrm{~A} / \mathrm{m}^{2}, k_{-}=32 \mathrm{~A} / \mathrm{m}^{2}$, $a=1.04 \times 10^{-10} \mathrm{~m}^{3} / \mathrm{C}, b_{+}=5.18 \times 10^{-11} \mathrm{~m}^{3} / \mathrm{C}, b_{-}=2.69 \times 10^{-11} \mathrm{~m}^{3} / \mathrm{C}, \gamma_{+}=9 \times 10^{-8} \mathrm{~m}$, $\gamma_{-}=6 \times 10^{-8} \mathrm{~m}, \mu^{m}=2.5 \times 10^{10} \mathrm{~N} / \mathrm{m}^{2}, \mu^{o}=1.24 \times 10^{10} \mathrm{~N} / \mathrm{m}^{2}, \nu^{m}=0.33, \nu^{o}=0.22$, $s_{11}^{+}=s_{22}^{+}=1.29 \times 10^{-11} \mathrm{~m}^{2} / \mathrm{N}, s_{11}^{-}=s_{22}^{-}=8.00 \times 10^{-12} \mathrm{~m}^{2} / \mathrm{N}, s_{13}^{+}=s_{31}^{+}=1.63 \times 10^{-11} \mathrm{~m}^{2} / \mathrm{N}$, $s_{13}^{-}=s_{31}^{-}=8.24 \times 10^{-12} \mathrm{~m}^{2} / \mathrm{N}, \alpha=-8 \times 10^{-9} \mathrm{~N} / \mathrm{V}^{2}$ and $\beta=5 \times 10^{-9} \mathrm{~N} / \mathrm{V}^{2}$.

The coefficients $\Sigma_{1,2}$ characterize the stress contributions to the basic state stability in this limit. In the absence of stress effects, the system can only yield long-wave instabilities which usually lead to the growth of irregular arrays of pores seen in other oxide materials such as titanium as well as AAO for certain parameter regimes [31, 32]. Taking typical electrochemical [43, 42, 41, 15, 44], electrostrictive [38] and elastic [45, 46, 44, 47] parameter values, we numerically obtain the roots of Eq. (4.50) and find that $\omega_{2}(q)<0$ for all values of $q$, and $\omega_{1}(q)$ will give the desired short-wave instability shown in Fig. 3 (parameters are listed in the caption). The wave number of the most rapidly growing mode for these parameter values is $q_{c} \approx 0.027 \mathrm{~nm}^{-1}$ which corresponds to pore spacings of about $230 \mathrm{~nm}$ and pore diameters of this order as well. Usually, systems which lack a reflection symmetry in the general solution vector will lead to the formation of hexagonal patterns near the onset of a short-wave instability [48, 49]. This can be explained by analyzing the system as its perturbations enter a weakly nonlinear regime, where the next order terms will be quadratic. In Fourier space, the hexagonal pattern is characterized by the superposition of three modes with wave vectors $\left(\mathbf{q}_{1}, \mathbf{q}_{2}, \mathbf{q}_{3}\right)$ of equal moduli such that $\mathbf{q}_{1}+\mathbf{q}_{2}+\mathbf{q}_{3}=0$. The quadratic nonlinearity produces the modes corresponding to the sum of two wave vectors in the annulus of instability about $|\mathbf{q}|=q_{c}$, as shown in Fig. 4. Thus, in the case of a hexagonal pattern, the modes produced by the quadratic nonlinearity will grow and be in resonance with the primary modes. This explains the ubiquity of hexagonal patterns in systems with quadratic nonlinearities exhibiting short-wave (Turing-type) instability.

We can also use the dispersion relation to calculate boundaries in parameter space indicating regions of stability and both short-wave and long-wave instabilities, which corre- 


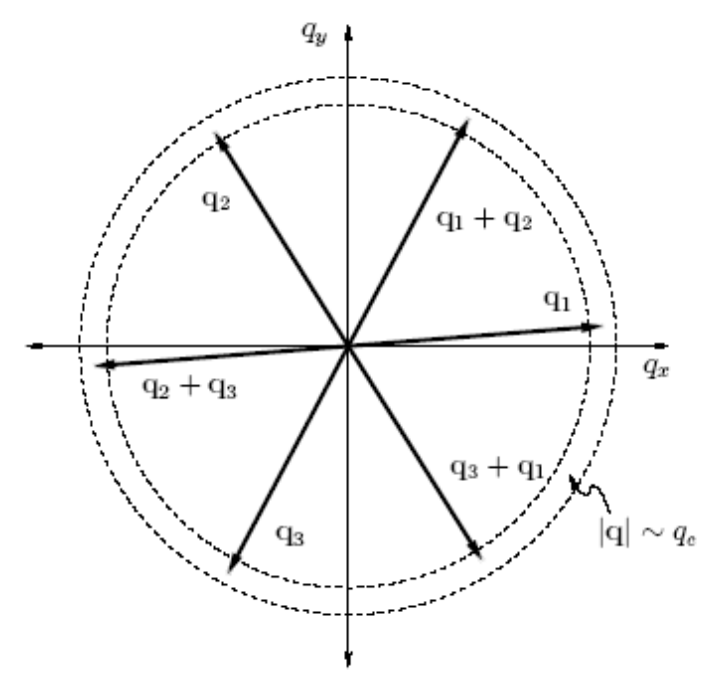

Figure 4: Schematic showing the quadratic resonance of hexagonal modes in the annulus of unstable wave vectors.

spond to barrier-type, ordered-porous and disordered-porous oxide layers respectively. These boundaries are determined by the system of equations

$$
C_{0}(q)=0, \quad \partial_{q} C_{0}(q)=0,
$$

and typical plots are presented in Fig. 5. Fig. 5(a) shows the phase diagram in the $(\alpha, \beta)$ parameter plane. One can see that the instabilities transition from long-wave to short-wave type to a damped regime as both $\alpha$ and $\beta$ increase. Fig. 5(b) shows the phase diagram in the parameter plane of the applied voltage and electrolyte $\mathrm{pH}$. One can see that as the $\mathrm{pH}$ is increased, the window for short-wave instability rapidly narrows, and lower voltages are required to obtain this instability. This diagram is similar to that obtained in [32].

We must also discuss the limitations of our model. We note that the dependence of the critical wave number, $q_{c}$, on the applied voltage seems to disagree with experimental evidence. While the dispersion relation predicts the critical wave number to increase with the voltage (i.e. a decrease in excited wavelengths), it has been observed that pore spacings and diameters increase linearly with the voltage $[15,23,43]$. In our model many effects have been neglected for simplicity, and while electrostriction appears to damp long-wave disturbances and lead to short-wave instability, the inclusion of additional mechanisms might yield the proper voltage dependence.

\section{Conclusion}

A mathematical model to describe the spontaneous self-assembly of nano-scale pores in AAO has been formulated. We have included the effects of electrostriction to the strains within 

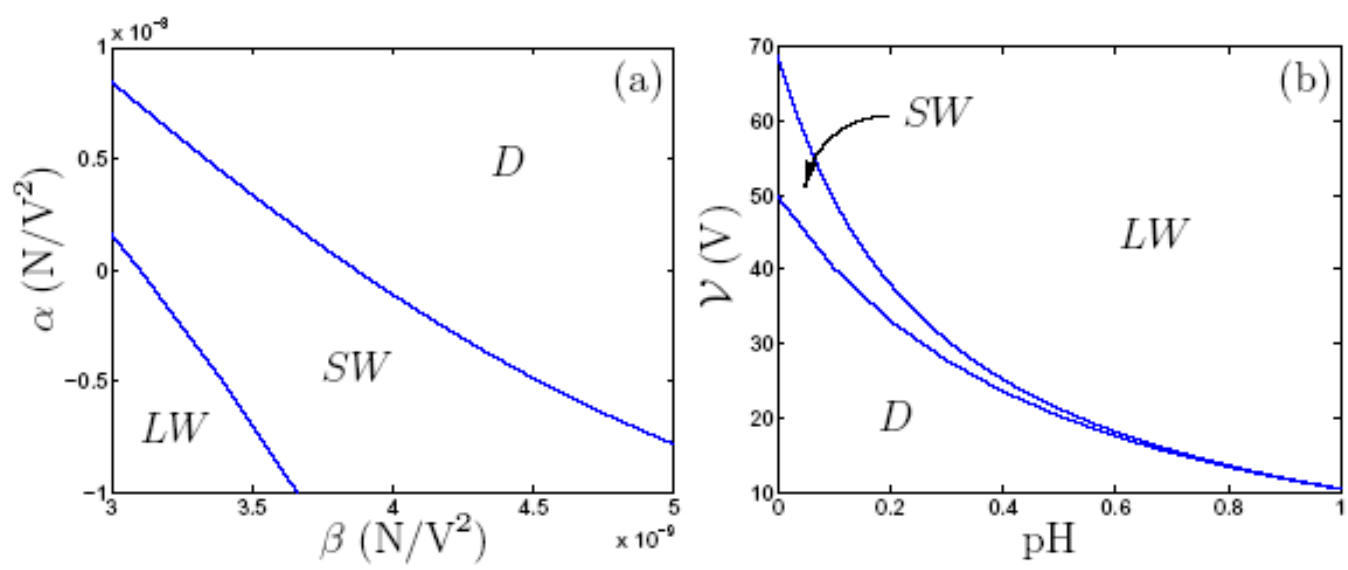

Figure 5: Phase diagrams indicating parameter regions of damped $(D)$, short-wave $(S W)$, and long-wave $(L W)$ instabilities. Left: $\alpha$ and $\beta$ varied with $\mathcal{V}=50 \mathrm{~V}$ and $\mathrm{pH}=0$. Right: $\mathcal{V}$ and $\mathrm{pH}$ varied with $\alpha=-8 \times 10^{-9} \mathrm{~N} / \mathrm{V}^{2}$ and $\beta=5 \times 10^{-9} \mathrm{~N} / \mathrm{V}^{2}$. Remaining parameters are the same as for Fig. 3.

the oxide layer and metal substrate along with the charge flux at the MO and OE interfaces described by the Butler-Volmer relations. A basic state solution of the system was calculated corresponding to an oxide layer of barrier type in which the oxidation of the metal substrate is balanced by the dissolution of the oxide into the electrolyte. The linear stability analysis of this state revealed that electrostrictive effects can induce the short-wave instability necessary for the hexagonal pattern selection observed in porous AAO. We have also presented phase diagrams which give parameter regimes for expected barrier-type, ordered-porous and disordered-porous oxide layers. We can hence conclude that electrostriction can act as a possible mechanism for the formation of hexagonally ordered pores in AAO.

\section{Acknowledgements}

This work was supported by the US NSF RTG grant \# DMS-0636574. 


\section{Appendix}

The elements of $\mathbf{Q}$ are listed as follows. The interfacial velocity conditions (4.38)-(4.40) give

$$
Q_{13}=-a \sigma_{0} E q, \quad Q_{1 k}=0 \forall k \neq 3
$$

and

$$
\begin{aligned}
Q_{21}= & 2 q\left(\nu^{o} \alpha+\left(1-2 \nu^{o}\right) \beta\right)\left(b_{+} S_{11}^{+}+b_{-} S_{11}^{-}+b_{+} S_{22}^{+}+b_{-} S_{22}^{-}\right) E^{2} \sinh (q L) \\
& +E\left(b_{+} A_{+}+b_{-} A_{-}\right) \cosh (q L), \\
Q_{22}= & i q\left(b_{+} S_{13}^{+}+b_{-} S_{13}^{-}+b_{+} S_{31}^{+}+b_{-} S_{31}^{-}\right) \bar{\sigma}_{11}^{o} \\
& -\left[\left(b_{+} A_{+}+b_{-} A_{-}\right) E-\left(b_{+} \Gamma_{+}+b_{-} \Gamma_{-}\right) q^{2}\right] \\
Q_{23}= & 2 q\left(\nu^{o} \alpha+\left(1-2 \nu^{o}\right) \beta\right)\left(b_{+} S_{11}^{+}+b_{-} S_{11}^{-}+b_{+} S_{22}^{+}+b_{-} S_{22}^{-}\right) E^{2} \cosh (q L) \\
& +\left(b_{+} A_{+}+b_{-} A_{-}\right) E \sinh (q L), \\
Q_{24}= & \left(b_{+} S_{11}^{+}+b_{-} S_{11}^{-}\right)\left[2 i q \mu^{o}\left(\frac{3-2 \nu^{o}}{3-4 \nu^{o}}\right) \cosh (q L)+\left(\frac{2 i q^{2} L \mu^{o}}{3-4 \nu^{o}}\right) \sinh (q L)\right] \\
& +\left(b_{+} S_{22}^{+}+b_{-} S_{22}^{-}\right)\left(\frac{4 i q \mu^{o} \nu^{o}}{3-4 \nu^{o}}\right) \cosh (q L), \\
Q_{25}= & \left(b_{+} S_{11}^{+}+b_{-} S_{11}^{-}\right)\left[\left(\frac{2 q^{2} L \mu^{o}}{3-4 \nu^{o}}\right) \cosh (q L)+\left(\frac{4 q \mu^{o} \nu^{o}}{3-4 \nu^{o}}\right) \sinh (q L)\right] \\
& +\left(b_{+} S_{22}^{+}+b_{-} S_{22}^{-}\right)\left(\frac{4 q \mu^{o} \nu^{o}}{3-4 \nu^{o}}\right) \sinh (q L), \\
Q_{26}= & \left(b_{+} S_{11}^{+}+b_{-} S_{11}^{-}\right)\left[2 i q \mu^{o}\left(\frac{3-2 \nu^{o}}{3-4 \nu^{o}}\right) \sinh (q L)+\left(\frac{2 i q^{2} L \mu^{o}}{3-4 \nu^{o}}\right) \cosh (q L)\right] \\
& +\left(b_{+} S_{22}^{+}+b_{-} S_{22}^{-}\right)\left(\frac{4 i q \mu^{o} \nu^{o}}{3-4 \nu^{o}}\right) \sinh (q L), \\
Q_{27}= & \left(b_{+} S_{11}^{+}+b_{-} S_{11}^{-}\right)\left[\left(\frac{2 q^{2} L \mu^{o}}{3-4 \nu^{o}}\right) \sinh (q L)+\left(\frac{4 q \mu^{o} \nu^{o}}{3-4 \nu^{o}}\right) \cosh (q L)\right] \\
& +\left(b_{+} S_{22}^{+}+b_{-} S_{22}^{-}\right)\left(\frac{4 q \mu^{o} \nu^{o}}{3-4 \nu^{o}}\right) \cosh (q L) . \\
& \\
\left.Q_{1}\right) & \\
& \\
&
\end{aligned}
$$

The charge flux (4.36)-(4.37) at the OE interface gives

$$
\begin{aligned}
Q_{31}= & 2 q\left(\nu^{o} \alpha+\left(1-2 \nu^{o}\right) \beta\right)\left(S_{11}^{+}+S_{11}^{-}+S_{22}^{+}+S_{22}^{-}\right) E^{2} \sinh (q L) \\
& +\sigma_{0} E q \sinh (q L)+E\left(A_{+}+A_{-}\right) \cosh (q L) \\
Q_{32}= & i q\left(S_{13}^{+}+S_{13}^{-}+S_{31}^{+}+S_{31}^{-}\right) \bar{\sigma}_{11}^{o}-\left(A_{+}+A_{-}\right) E+\left(\Gamma_{+}+\Gamma_{-}\right) q^{2},
\end{aligned}
$$




$$
\begin{aligned}
Q_{33}= & 2 q\left(\nu^{o} \alpha+\left(1-2 \nu^{o}\right) \beta\right)\left(S_{11}^{+}+S_{11}^{-}+S_{22}^{+}+S_{22}^{-}\right) E^{2} \cosh (q L) \\
& +\sigma_{0} E q \cosh (q L)+\left(A_{+}+A_{-}\right) E \sinh (q L), \\
Q_{34}= & \left(S_{11}^{+}+S_{11}^{-}\right)\left[2 i q \mu^{o}\left(\frac{3-2 \nu^{o}}{3-4 \nu^{o}}\right) \cosh (q L)+\left(\frac{2 i q^{2} L \mu^{o}}{3-4 \nu^{o}}\right) \sinh (q L)\right] \\
& +\left(S_{22}^{+}+S_{22}^{-}\right)\left(\frac{4 i q \mu^{o} \nu^{o}}{3-4 \nu^{o}}\right) \cosh (q L), \\
Q_{35}= & \left(S_{11}^{+}+S_{11}^{-}\right)\left[\left(\frac{2 q^{2} L \mu^{o}}{3-4 \nu^{o}}\right) \cosh (q L)+\left(\frac{4 q \mu^{o} \nu^{o}}{3-4 \nu^{o}}\right) \sinh (q L)\right] \\
& +\left(S_{22}^{+}+S_{22}^{-}\right)\left(\frac{4 q \mu^{o} \nu^{o}}{3-4 \nu^{o}}\right) \sinh (q L), \\
Q_{36}= & \left(S_{11}^{+}+S_{11}^{-}\right)\left[2 i q \mu^{o}\left(\frac{3-2 \nu^{o}}{3-4 \nu^{o}}\right) \sinh (q L)+\left(\frac{2 i q^{2} L \mu^{o}}{3-4 \nu^{o}}\right) \cosh (q L)\right] \\
& +\left(S_{22}^{+}+S_{22}^{-}\right)\left(\frac{4 i q \mu^{o} \nu^{o}}{3-4 \nu^{o}}\right) \sinh (q L), \\
Q_{37}= & \left(S_{11}^{+}+S_{11}^{-}\right)\left[\left(\frac{2 q^{2} L \mu^{o}}{3-4 \nu^{o}}\right) \sinh (q L)+\left(\frac{4 q \mu^{o} \nu^{o}}{3-4 \nu^{o}}\right) \cosh (q L)\right] \\
& +\left(S_{22}^{+}+S_{22}^{-}\right)\left(\frac{4 q \mu^{o} \nu^{o}}{3-4 \nu^{o}}\right) \cosh (q L) .
\end{aligned}
$$

The stress conditions (4.32) at the MO interface give

$$
\begin{aligned}
Q_{41}= & 2 i q \mu^{m}\left(\frac{1-2 \nu^{m}}{3-4 \nu^{m}}\right)\left[\bar{u}_{33}^{o}+\frac{E^{2}}{\mu^{o}}\left(1-2 \nu^{o}\right)(\alpha-2 \beta)\right] \\
& +i q\left(\frac{1-2 \nu^{o}}{1-\nu^{o}}\right)\left(\nu^{o} \alpha+\left(1-2 \nu^{o}\right) \beta\right) E^{2}, \\
Q_{42}= & Q_{43}=0, \quad Q_{44}=4 q \mu^{m}\left(\frac{1-\nu^{m}}{3-4 \nu^{m}}\right), \\
Q_{45}= & 2 i q \mu^{m}\left(\frac{1-2 \nu^{m}}{3-4 \nu^{m}}\right)-2 i q \mu^{o}\left(\frac{1-2 \nu^{o}}{3-4 \nu^{o}}\right), \\
Q_{46}= & -4 q \mu^{o}\left(\frac{1-\nu^{o}}{3-4 \nu^{o}}\right), \quad Q_{47}=0,
\end{aligned}
$$

and

$$
\begin{aligned}
& Q_{51}=4 q \mu^{m}\left(\frac{1-\nu^{m}}{3-4 \nu^{m}}\right)\left[\bar{u}_{33}^{o}+\frac{E^{2}}{\mu^{o}}\left(1-2 \nu^{o}\right)(\alpha-2 \beta)\right] \\
& Q_{52}=0, \quad Q_{53}=2 q\left(\nu^{o} \alpha+\left(1-2 \nu^{o}\right) \beta\right) E^{2} \\
& Q_{54}=-Q_{45}, \quad Q_{55}=Q_{44}, \quad Q_{56}=0, \quad Q_{57}=Q_{46} .
\end{aligned}
$$


Finally, the stress conditions (4.33) at the OE interface give

$$
\begin{aligned}
& Q_{61}=-2 i q\left(\nu^{o} \alpha+\left(1-2 \nu^{o}\right) \beta\right) E^{2} \cosh (q L), \quad Q_{62}=-i q \bar{\sigma}_{11}^{o}, \\
& Q_{63}=-2 i q\left(\nu^{o} \alpha+\left(1-2 \nu^{o}\right) \beta\right) E^{2} \sinh (q L), \\
& Q_{64}=4 q \mu^{o}\left(\frac{1-\nu^{o}}{3-4 \nu^{o}}\right) \sinh (q L)+\left(\frac{2 q^{2} L \mu^{o}}{3-4 \nu^{o}}\right) \cosh (q L), \\
& Q_{65}=2 i q \mu^{o}\left(\frac{1-2 \nu^{o}}{3-4 \nu^{o}}\right) \cosh (q L)-\left(\frac{2 i q^{2} L \mu^{o}}{3-4 \nu^{o}}\right) \sinh (q L), \\
& Q_{66}=4 q \mu^{o}\left(\frac{1-\nu^{o}}{3-4 \nu^{o}}\right) \cosh (q L)+\left(\frac{2 q^{2} L \mu^{o}}{3-4 \nu^{o}}\right) \sinh (q L), \\
& Q_{67}=2 i q \mu^{o}\left(\frac{1-2 \nu^{o}}{3-4 \nu^{o}}\right) \sinh (q L)-\left(\frac{2 i q^{2} L \mu^{o}}{3-4 \nu^{o}}\right) \cosh (q L),
\end{aligned}
$$

and

$$
\begin{aligned}
& Q_{71}=-i Q_{63}, \quad Q_{72}=0, \quad Q_{73}=-i Q_{61}, \\
& Q_{74}=-2 i q \mu^{o}\left(\frac{1-2 \nu^{o}}{3-4 \nu^{o}}\right) \cosh (q L)-\left(\frac{2 i q^{2} L \mu^{o}}{3-4 \nu^{o}}\right) \sinh (q L), \\
& Q_{75}=4 q \mu^{o}\left(\frac{1-\nu^{o}}{3-4 \nu^{o}}\right) \sinh (q L)-\left(\frac{2 q^{2} L \mu^{o}}{3-4 \nu^{o}}\right) \cosh (q L), \\
& Q_{76}=-2 i q \mu^{o}\left(\frac{1-2 \nu^{o}}{3-4 \nu^{o}}\right) \sinh (q L)-\left(\frac{2 i q^{2} L \mu^{o}}{3-4 \nu^{o}}\right) \cosh (q L), \\
& Q_{77}=4 q \mu^{o}\left(\frac{1-\nu^{o}}{3-4 \nu^{o}}\right) \cosh (q L)-\left(\frac{2 q^{2} L \mu^{o}}{3-4 \nu^{o}}\right) \sinh (q L) .
\end{aligned}
$$

\section{References}

[1] S. Wernick, R.Pinner, P. G. Sheasby. The surface treatment and finishing of aluminum and its alloys. (ASM International, Metals Park, 1987).

[2] J. P. O'Sullivan, G. C. Wood. The morphology and mechanism of formation of porous anodic films on aluminum. Proc. R. Soc. London, Ser. A, 317 (1970), 511-543.

[3] G. E. Thompson. Porous anodic alumina: fabrication, characterization and applications. Thin Solid Films, 297 (1997), 192-201.

[4] R. Beranek, H. Hildebrand, P. Schmuki. Self-Organized Porous Titanium Oxide Prepared in $\mathrm{H}_{2} \mathrm{SO}_{4} / \mathrm{HF}$ Electrolytes. Electrochem. Solid-State Lett., 6 (2003), B12-B14.

[5] D. Gong, C. A. Grimes, O. K. Varghese, W. C. Hu, R. S. Singh, Z. Chen, E. C. Dickey. Titanium oxide nanotube arrays prepared by anodic oxidation. J. Mater. Res., 16 (2001), 3331-3334. 
[6] J. M. Macak, H. Tsuchiya, P. Schmuki. High-aspect-ratio TiO $\mathrm{T}_{2}$ nanotubes by anodization of titanium. Angew. Chem. Int. Ed., 44 (2005), 2100-2102.

[7] H. Tsuchiya, J. M. Macak, A. Ghicov, L. Taveira, P. Schmuki. Self-organized porous $\mathrm{TiO}_{2}$ and $\mathrm{ZrO}_{2}$ produced by anodization. Corr. Sci., 47 (2005), 3324-3335.

[8] J. Choi, J. H. Lim, S. C. Lim, J. H. Chang, K. J. Kim, M. A. Cho. Porous niobium oxide films prepared by anodization in $\mathrm{HF} / \mathrm{H}_{3} \mathrm{PO}_{4}$. Electrochim. Acta, 51 (2006), 5502-5507.

[9] I. V. Sieber, P. Schmuki. Porous tantalum oxide prepared by electrochemical anodic oxidation. J. Electrochem. Soc., 152 (2005), C639-C644.

[10] H. Tsuchiya, J. M. Macak, I. Sieber, L. Taveira, A. Ghicov, K. Sirotna, P. Schmuki. Selforganized porous $\mathrm{WO}_{3}$ formed in NaF electrolytes. Electrochem. Commun., 7 (2005), 295-298.

[11] H. C. Shin, J. Dong, M. L. Liu. Porous tin oxides prepared using an anodic oxidation process. Adv. Mater. (Weinheim, Ger.), 16 (2004), 237-240.

[12] H. Masuda, K. Fukuda. Ordered Metal Nanohole Arrays Made by a Two-Step Replication of Honeycomb Structures of Anodic Alumina. Science, 268 (1995), 1466-1468.

[13] H. Masuda, H. Yamada, M. Satoh, H. Asoh, M. Nakao, T. Tamamura. Highly ordered nanochannel-array architecture in anodic alumina. Appl. Phys. Lett., 71 (1997), 27702772.

[14] A. P. Li, F. Muller, A. Birner, K. Nielsch, U. Gosale. Hexagonal pore arrays with a 50-420 $\mathrm{nm}$ interpore distance formed by self-organization in anodic alumina. J. Appl. Phys., 84 (1998), 6023-6026.

[15] F. Y. Li, L. Zhang, R. M. Metzger. On the growth of highly ordered pores in anodized aluminum oxide. Chem. Mater., 10 (1998), 2470-2480.

[16] A. Bai, C.-C. Hu, Y.-F. Yang, C.-C. Lin. Pore diameter control of anodic aluminum oxide with ordered array of nanopores. Electrochem. Acta, 53 (2008), 2258-2264.

[17] G. E. Thompson, G. C. Wood. Anodic films on aluminium. Treatise on Mater. Sci. and Tech., 23 (1983), 205-329.

[18] A. Huczko. Template-based synthesis of nanomaterials. Appl. Phys. A: Mater. Sci. Process., 70 (2000), 365-376.

[19] J. Choi, Y. Luo R. B. Wehrspohn, R. Hillebrand, J. Schilling, U. Gosele. Perfect twodimensional porous alumina photonic crystals with duplex oxide layers. J. Appl. Phys., 94 (2003), 4757-4762. 
[20] G. S. Cheng, M. Moskovits. A highly regular two-dimensional array of an quantum dots deposited in a periodically nanoporous GaAs epitaxial layer. Adv. Mater. (Weinheim, Ger.), 14 (2002), 1567-1570.

[21] G. Schmid. Materials in nanoporous alumina. J. Mater. Chem., 12 (2002), 1231-1238.

[22] H. Asoh, K. Nishio, M. Nakao, T. Tamamura, H. Masuda. Conditions for fabrication of ideally ordered anodic porous alumina using pretextured Al. J. Electrochem. Soc., 148 (2001), B152-B156.

[23] V. P. Parkhutic, V. I. Shershulsky, Theoretical modelling of porous oxide. J. Phys. D, 25 (1992), 1258-1263.

[24] J. E. Houser, K. R. Herbert. Modeling the potential distribution in porous anodic alumina films during steady-state growth. J. Electrochem. Soc., 153 (2006), B566-B573.

[25] S. K. Thamida, H. C. Chang. Nanoscale pore formation dynamics during aluminum anodization. Chaos, 12 (2002), 240-251.

[26] V. V. Yuzhakov, H. C. Chang, A. E. Miller. Pattern formation during electropolishing. Phys. Rev. B, 56 (1997), 12608-12624.

[27] M. Sheintuch, Y. Smagina. Nanopore formation dynamics during aluminum anodization. Physica D, 226 (2007), 95-105.

[28] W. Guo, D. Johnson. Role of interfacial energy during pattern formation of electropolishing. Phys. Rev. B, 67 (2003), 075411.

[29] F. Q. Yang. Stability of the metal-oxide interface during anodization of aluminum. Electrochem. Solid State Lett., 9 (2006), C44-C47.

[30] C. Sample, A. A. Golovin. Formation of porous metal oxides in the anodization process. Phys. Rev. E, 74 (2006), 041606.

[31] G. K. Singh, A. A. Golovin, I. S. Aranson, V. M. Vinokur. Formation of nanoscale pore arrays during anodization of aluminum. Europhys. Lett., 70 (2005), 836-842.

[32] G. K. Singh, A. A. Golovin, I. S, Aranson. Formation of self-organized nanoscale porous structures in anodic aluminum oxide. Phys. Rev. B, 73 (2006), 205422.

[33] A. L. Friedman, D. Brittain, L. Menon. Roles of $p H$ and acid type in the anodic growth of porous alumina. J. Chem. Phys., 127 (2007), 154717.

[34] A. L. Friedman, L. Menon. Dendritic-pattern formation in anodic aluminum oxide templates. J. Appl. Phys., 101 (2007), 084310.

[35] Z. Wu, C. Richter, L. Menon. A study of anodization process during pore formation in nanoporous alumina templates. J. Electrochem. Soc., 154 (2007), E8-E12. 
[36] S. H. Park, Y. Lee, J. K. Lee, K. B. Kim. The formation of porous anodic aluminum oxide confined in mu m-size contact and trench patterns. Electrochem. Solid State Lett., 9 (2006), D31-D33.

[37] K. S. Raja, M. Misra, K. Paramguru. Formation of self-ordered nano-tubular structure of anodic oxide layer on titanium. Electrochem. Acta, 51 (2005), 154-165.

[38] R. Yimnirun, P. J. Moses, R. J. Meyer, R. E. Newnham. Electrostrictive strain in lowpermittivity dielectrics. J. Electroceram., 8 (2002), 87-98.

[39] L. D. Landau, E. M. Lifshitz. Electrodynamics of continuous media. Pergamon Press, Oxford, 1960.

[40] J. O’M. Bockris, A. K. N. Reddy. Modern electrochemistry, Plenum Press, New York, 1970.

[41] O. Jessensky, F. Muller, U. Gosele. Self-organized formation of hexagonal pore arrays in anodic alumina. Appl. Phys. Lett., 72 (1998), 1173-1175.

[42] J. W. Diggle. Oxides and anodic films. Marcel Dekker, New York, 1973, Vol. 2.

[43] G. Paolini, M. Masoero, F. Sacchi, W. Paganell. An Investigation of porous anodic oxide films on aluminum by comparative adsorption gravimetric and electronoptical measurements. J. Electrochem. Soc., 112 (1965), 32-38.

[44] A. Valance. Porous silicon formation - stability analysis of the silicon-electrolyte interface. Phys. Rev. B, 52 (1995), 8323-8336.

[45] CRC Materials Science and Engineering Handbook, 3rd ed., edited by J. F. Schackelford and W. Alexander, CRC Press, Boca Raton, 2001.

[46] Metals Handbook, 2nd ed., edited by J. R. Davis ASM International, Materials Park, $\mathrm{OH}, 1998$.

[47] G. Grinstein, Y. Tu, J. Tersoff. Stability of solid state reaction fronts. Phys. Rev. Lett., 81 (1998), 2490-2493.

[48] M. C. Cross, P. C. Hohenberg. Pattern-formation outside of equilibrium. Rev. Mod. Phys., 65 (1993), 851-1112.

[49] D. Walgraef. Spatio-temporal pattern formation with examples from physics, chemistry and materials science. Springer-Verlag, Berlin, 1997. 